\title{
Search for the Star-Planet Interaction
}

\author{
Tereza Krejčová ${ }^{1}$, Ján Budaj ${ }^{2}$ and Július Koza ${ }^{2}$ \\ ${ }^{1}$ Dept. of Theoretical Physics and Astrophysics, Masaryk University, \\ Brno, Czech Republic, email: terak@physics.muni.cz \\ ${ }^{2}$ Astronomical Institute, Tatranská Lomnica, Slovak Republic, email: budaj@ta3.sk
}

\begin{abstract}
We analyse the chromospherical activity of stars with extrasolar planets and search for a possible correlation between the equivalent width of the core of the Ca II K line and orbital parameters of the planet. We found statistically significant evidence that the equivalent width of the Ca II K line reversal, which originates in the stellar chromosphere, depends on the orbital period $P_{\text {orb }}$ of the exoplanet. Planets orbiting stars with $T_{\text {eff }}<5500 \mathrm{~K}$ and with $P_{\text {orb }}<20$ days generally have much stronger emission than planets at similar temperatures but at longer orbital periods. $P_{\text {orb }}=20$ days marks a sudden change in behaviour, which might be associated with a qualitative change in the star-planet interaction.
\end{abstract}

Keywords. Ca II K line, exoplanet, star-planet interaction.

\section{Introduction}

The question of the possible existence of star-planet interactions is currently studied in many ways. Based on the observations in the optical region Shkolnik et al. (2005, 2008) discovered the planetary induced variability in the cores of Ca II H \& K, H $\alpha$ and Ca II IR triplet in a few planet hosting stars. Knutson et al. (2010) found a correlation between the chromospheric activity of the star and presence of the stratosphere on the planet. Consequently, Hartman (2010) found a correlation between the surface gravity of Hot Jupiters and the stellar activity. Recently Canto Martins et al. (2011) searched for correlation between planetary parameters and the $\log R_{\mathrm{HK}}^{\prime}$ parameter but didn't reveal any convincing proof for such a phenomenon.

\section{Observation \& Statistical Analysis}

We used the FEROS instrument on the $2.2 \mathrm{~m}$ ESO/MPG telescope to obtain spectra of several stars (HD 179949, HD 212301, HD 149143 and Wasp-18) with close-in exoplanets. We also used the publicly available spectra from the HIRES spectrograph archive. Subsequently we measured the equivalent width of the central reversal in the core of Ca II K.

In the first case we divided our data sample into two groups according to the semimajor axis $(a \leqslant 0.15$ and $a>0.15 \mathrm{AU})$. Figure 1 (left-top) shows the dependence of equivalent width on the effective temperature of the star. Subsequently, we performed two statistical tests - Student's t-test and the Kolmogorov-Smirnov test to determine whether the two groups originate from the same population. The resulting probability is a function of temperature and is plotted in the lower part of Figure 1. The tests show that the difference between the two samples is significant for $T_{\text {eff }} \leqslant 5500 \mathrm{~K}$. It means that stars with lower temperatures and with planets on closer orbits show more activity as measured in the core of Ca II K line. 

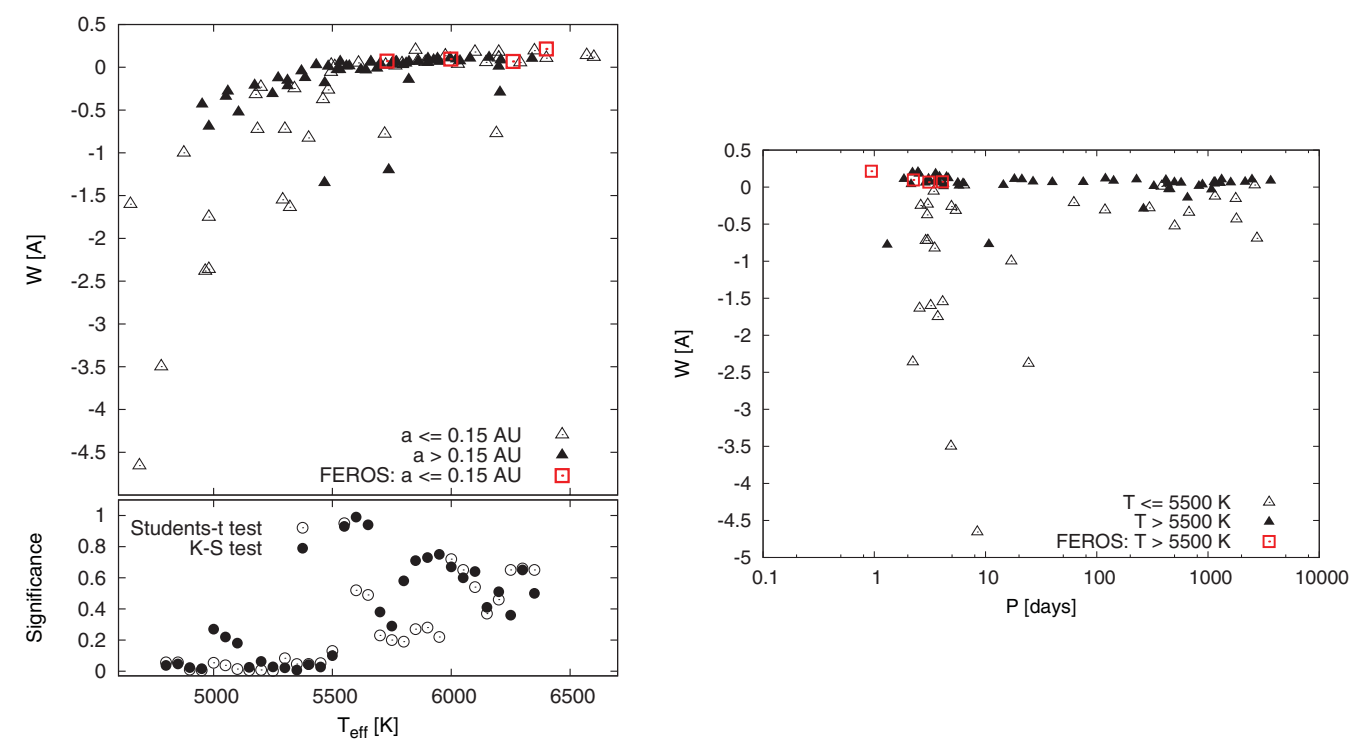

Figure 1. Left Top: Dependence of the equivalent width of Ca II K reversal on the temperature of the parent star. Empty triangles are exoplanetary systems with $a \leqslant 0.15 \mathrm{AU}$, full triangles are systems with $a>0.15$ AU. Bottom: Statistical Student's t-test (empty circles) and Kolmogorov-Smirnov test (full circles). Red squares are data from FEROS. Right Dependence of the equivalent width of Ca II K on the orbital period. Empty triangles are exoplanetary systems with $T \leqslant 5500 \mathrm{~K}$, full triangles are systems with $T>5500 \mathrm{~K}$ and red squares are data from FEROS.

In the second case, we group the data according to the effective temperature of the parent star $\left(T_{\text {eff }} \leqslant 5500 \mathrm{~K}\right.$ and $\left.T_{\text {eff }}>5500 \mathrm{~K}\right)$ and plot the equivalent width of the Ca II K line reversal as a function of the orbital period (Figure 1-right).

\section{Acknowledgements}

This work has been supported by grant GA ČR GD205/08/H005, VEGA 2/0078/10, VEGA 2/0074/09, VEGA 2/0094/11 and the National scholarship programme of Slovak Republic. This research has made use of the Keck Observatory Archive (KOA), which is operated by the W. M. Keck Observatory and the NASA Exoplanet Science Institute (NExScI), under contract with the National Aeronautics and Space Administration. We want to thank Tomáš Henych for fruitful discussions.

\section{References}

Canto Martins, B. L., Das Chagas, M. L., Alves, S., et al., 2011, A $\& A$, 530, A73

Hartman, J. D. 2010, ApJ, 717, L138

Knutson, H. A., Howard, A. W., \& Isaacson, H. 2010, ApJ, 720, 1569

Shkolnik, E., Bohlender, D. A., Walker, G. A. H., \& Collier Cameron, A. 2008, ApJ, 676, 628

Shkolnik, E., Walker, G. A. H., Bohlender, D. A., Gu, P., \& Kurster, M. 2005, ApJ, 622, 1075 